\title{
The impact of selection using residual average daily gain and marbling EPDs on growth, performance, and carcass traits in Angus steers ${ }^{1}$
}

\author{
Rachael A. Detweiler, ${ }^{\dagger}$ T. Dean Pringle, ${ }^{\dagger}$ Romdhane Rekaya, ${ }^{\dagger}$ Jonathan B. Wells, ${ }^{\ddagger}$ and Jacob R. Segers ${ }^{\|, 2}$ \\ ${ }^{\dagger}$ Department of Animal and Dairy Science, The University of Georgia, Athens, GA 30605; ${ }^{\ddagger}$ Oglethorpe Feed and Seed, \\ Crawford, GA 30630; and "Department of Animal and Dairy Science, The University of Georgia, Tifton, GA 31793
}

\begin{abstract}
One hundred ninety-one Angus steers $($ age $=546 \mathrm{~d} \pm 33.5 ; \mathrm{BW}=36.4 \mathrm{~kg} \pm 4.2$ ), sired by bulls divergently selected for feed efficiency over a 3-yr period, were used to compare growth, efficiency, body composition, and carcass characteristics. Selected Angus sires were either high (Hi) or low (Lo) for residual average daily gain (RADG) expected progeny differences (EPDs) and either high (Hi) or low (AVG; breed average) for marbling (MARB) EPDs. Steer weight and body composition, via ultrasound, were measured at weaning (205 d) and at $1 \mathrm{yr}(365 \mathrm{~d})$ of age. Steers entered the feedlot at $454 \mathrm{~d}$ of age and completed a 70-d GROWSAFE BEEF System evaluation to determine DMI, ADG, and residual feed intake (RFI). Steers were then slaughtered as they reached a backfat thickness of $1.3 \mathrm{~cm}$. Carcasses were chilled for $48 \mathrm{~h}$ at $2{ }^{\circ} \mathrm{C}$, ribbed, and USDA yield and quality grade data were collected. The right side of the carcass was fabricated and primal and subprimal weights were collected. A $2.5-\mathrm{cm}$ longissimus steak was removed, vacuum-packaged, aged for $14 \mathrm{~d}$, and frozen for slice shear force determination. Additionally, a $1.3-\mathrm{cm}$ longissimus steak was removed from year 3 steers for proximate analysis. The GLM procedure of SAS was
\end{abstract}

used and the main effects of RADG and MARB and their interaction were tested by the error term, SIRE(RADG*MARB). Year was evaluated as a replicate. Weight, ultrasound backfat, and REA were increased $(P \leq 0.05)$ at weaning in the $\mathrm{Hi}$ compared with AVG MARB steers. Feed efficiency, measured by RFI, was improved $(P=0.05)$ in the Hi RADG steers compared with Lo RADG steers. Slaughter weight and $\mathrm{HCW}$ were heavier $(P \leq 0.03)$ in the Hi RADG steers compared with Lo RADG steers. An interaction $(P=0.05)$ between RADG and MARB selection was found for marbling score, steers selected for Lo RADG and Hi MARB had greater marbling scores than all other groups. Longissimus proximate composition from year 3 showed that lipid content was greater $(P<0.01)$ in the Hi MARB and Lo RADG groups compared with the AVG MARB and Hi RADG groups, respectively. These findings suggest that selection using RADG or MARB EPDs has minimal impact on carcass yield. However, positive selection pressure placed on these breeding values can potentially improve efficiency and carcass quality. Lastly, it appears that improvements in feed efficiency can be obtained without negatively affecting beef carcass merit, especially USDA quality grade.

Key words: Angus, carcass, marbling, residual average daily gain

(C) The Author $(s)$ 2019. Published by Oxford University Press on behalf of the American Society of Animal Science. All rights reserved. For permissions, please e-mail: journals.permissions@oup.com.

J. Anim. Sci. 2019.97:2450-2459

doi: $10.1093 / \mathrm{jas} / \mathrm{skz} 124$

\footnotetext{
${ }^{1}$ Special thanks to Mr. Steve Whitmire, owner of Ridgefield Farm and Brasastown Beef, for the use of their feedlot and meat plant; to the crew at the Northwest Georgia Research and Education Center for their management of the cow herd; and to the Georgia Agricultural Commod-
}

ity Commission for Beef for partial financial support of this project.

${ }^{2}$ Corresponding author: jacobs@uga.edu

Received December 5, 2018.

Accepted March 9, 2019. 


\section{INTRODUCTION}

Approximately $65 \%$ of the total farm expenditures in beef cattle operations are associated with feed cost (Lancaster et al., 2009). Therefore, producers must be able to balance output (growth) with feed costs during the bull selection process (Rolf et al., 2010). Until recently, there was no genetic predictor available to beef producers which would allow them to select for improved efficiency in their herds. However, the American Angus Association developed the residual average daily gain (RADG) expected progeny difference (EPD) in 2010. This EPD incorporates calf weaning weight, postweaning gain, ultrasound subcutaneous fat thickness, and genomic dry-matter intake (Northcutt, 2010; MacNeil et al., 2011; Nielsen et al., 2013). The RADG EPD assesses the genetic value of an animal for postweaning average daily gain given a constant amount of feed. It is expressed in pounds per day where a higher value of RADG EPD indicates a more feed efficient (low residual feed intake [RFI]) animal, and a lower value is indicative of a less feed-efficient (high RFI) animal. The objective of this study was to compare growth performance, carcass characteristics, and feed efficiency in steers sired by Angus bulls that were divergently selected, based on their RADG and marbling (MARB) EPDs.

\section{MATERIALS AND METHODS}

\section{Experimental Design}

All animals used in this study were managed according to the guidelines set by the University of Georgia Animal Care and Use Committee guidelines (AUP \#A2012 11-006-R1). For 3 yr, commercial Angus cows from the Northwest Georgia Research and Education Center in Calhoun, GA have been assigned for breeding to Angus sires to determine the effect of divergent selection for RADG and MARB on growth, feed efficiency, and carcass traits in Angus steers. Sire EPD's for RADG and MARB were organized into a $2 \times 2$ factorial arrangement of treatments. Sires were selected to have either a high or low RADG EPD and within the RADG lines, half of the sires were selected to have high MARB EPDs, whereas the remaining sires were selected to have near breed average MARB EPDs.

\section{Sire Selection}

Bulls in this study were selected each year in January 2013, 2014, and 2015, from the American
Angus Association's Sire Evaluation Report. At the time of selection, the high RADG bulls ranked in the top 5 th percentile $(+0.27$ or higher) and the low RADG bulls ranked in the 95 th percentile $(+0.10$ or lower) with accuracies of 0.40 or higher. Accuracies for the RADG EPD are relatively low due to the limited data used for their estimation. Bulls were first sorted into low and high RADG EPD lines. Within each line, bulls were grouped into high MARB EPD (top 5th percentile; EPD > 0.94) and low MARB EPD (around 50th percentile; EPD $\approx$ 0.46) groups. More divergent MARB EPD populations were not selected so that carcass quality was not overly compromised in the cow herd. Bulls across the RADG EPD lines were also selected to be comparable in growth and carcass traits (other than MARB EPDs) and to minimize the potential for inbreeding. Two bulls (one from each RADG EPD line) were used across subsequent years to assure connectivity of data collected across years and allow a more robust statistical inference. The two main sire selections (RADG and MARB) were organized into a $2 \times 2$ factorial arrangement of treatments that contained 2 Angus sires in each of the following treatments: high RADG, high MARB (Hi/Hi); high RADG, average MARB (Hi/Avg); low RADG, high MARB (Lo/Hi); and low RADG, average MARB (Lo/Avg) for a total of 8 bulls each breeding season in this experiment.

\section{Semen Procurement and Breeding}

After bulls were selected for the breeding season, the owners of the bulls were contacted and semen was procured. Next, cows and heifers at the Northwest Georgia Research and Education Center in Calhoun, GA were synchronized in March of 2013, 2014, and 2015 using the Select Synch + CIDR \& TAI protocol. Approximately 30 cows were bred to each bull each year.

\section{Calf Processing and Weaning}

A total of 191 steer calves were used in this study: year $1(n=68)$, year $2(n=63)$, and year $3(n=60)$. Each calving season, male calves were surgically castrated, vaccinated with BoviShield Gold One Shot (Zoetis, Florham Park, NJ) and Ultra Choice 7 (Zoetis, Florham Park, NJ), then Dectomax (Zoetis, FlorhaM Park, NJ) pour-on was used for deworming. At weaning, weights were recorded and carcass ultrasound data were collected. Ribeye area, 12th-rib backfat thickness, and intramuscular fat percentage were collected using an Aloka 500-V 
ultrasound unit with a 17.2 a.m., 3.5 MHz linear probe (Corometrics Medical Systems, Wallingford, CT). Data collected were then interpreted using Beef Information Analysis Pro Plus software (Designer Genes USA, Harrison, AR). Steers from calving seasons were fence line weaned.

\section{Calf Backgrounding and Yearling}

Year one weaned calves were backgrounded on fescue (Festuca arundinacea) and annual ryegrass (Lolium multiflorum) pasture with Russell Bermudagrass (Cynodon dactylon) hay and baleage. Calves also received $3.6 \mathrm{~kg} / \mathrm{hd} / \mathrm{d}$ of a coproductbased supplement containing 50\% corn gluten feed and 50\% soybean hulls. Calves for years two and three were shipped at weaning to Ridgefield Farm L.L.C. in Brasstown, North Carolina and backgrounded on a blended corn silage supplement containing soy hull pellets, minerals, and dry distillers grain from late September until February (Table 1). At $1 \mathrm{yr}(366.8 \pm 37.9 \mathrm{~d})$ of age, BW was recorded and real-time carcass ultrasound data were collected. Ribeye area, 12th-rib backfat thickness, and intramuscular fat percentage images were collected using an Aloka 500-V ultrasound unit with a 17.2 a.m., 3.5 MHz linear probe (Corometrics Medical Systems, Wallingford, CT). Images were then interpreted using Beef Information Analysis Pro Plus software (Designer Genes USA, Harrison, AR).

Table 1. Formulation and calculated chemical composition of finishing diet fed to steers from cows breed to Angus bulls with high (Hi) or low (Lo) residual average daily gain (RADG) and high (Hi) or breed average (AVG) marbling (MARB) EPDs

\begin{tabular}{lc}
\hline \hline Ingredients $^{1}$ & $\%$ As-fed basis \\
\hline Corn Silage & 25.0 \\
Cracked Corn & 37.9 \\
Soy Hull Pellets & 6.5 \\
Dry Distillers Grain & 27.0 \\
Beef Grower ${ }^{2}$ & 1.8 \\
Chemical Composition ${ }^{3}$ & \\
Crude Protein, \% & 17.49 \\
Fat, \% & 5.89 \\
Neutral Detergent Fiber, \% & 25.01 \\
Total Digestible Nutrients, \% & 81.16 \\
Net Energy Maintenance, Mcal/kg & 1.98 \\
Net Energy Gain, Mcal/kg & 1.33 \\
Calcium, \% & 0.81 \\
Phosphorous, \% & 0.52 \\
\hline
\end{tabular}

${ }^{1}$ Ingredients included in steer finishing ration on as fed basis (\%).

${ }^{2}$ Protein suppelement with vitamin and mineral premix.

${ }^{3}$ Calculated analysis (DM basis, \%), nutrient concentration.

\section{Efficiency Evaluation}

After yearling ultrasound, year one steers were then shipped to Ridgefield Farm L.L.C. in Brasstown, North Carolina for finishing. Steers were allowed a 14-d acclimation period where they were given ad libidum access to a grain-based finishing diet (Table 1). Cattle were moved to the GROWSAFE BEEF System (GrowSafe Systems Ltd., Airdrie, Alberta, Canada) bunks and feed intake was measured over a 70-d period using the GROWSAFE BEEF System. Steer weights were collected at the beginning, midpoint, and end of the 70-d feed test. On test and off test weights were collected at the same time on two subsequent days and the average is reported to correct of differences in rumen fill. Ultrasound data were collected at the beginning and end of the 70-d feed test. A linear regression of midpoint metabolic body weight and average daily gain over the 70-d test was used to predict intake. Residual feed intake was then calculated by the following formula:

$$
\text { RFI }=\text { Actual Intake Predicted Intake. }
$$

\section{Harvest and Grading}

Steers were slaughtered at approximately $1.3 \mathrm{~cm}$ of backfat. Steers were slaughtered at Waldrop's Meat Processing in Ellijay, GA, a USDA-inspected slaughter facility. Steers were held overnight at the plant with access to water and slaughtered the following morning under USDA inspection. Carcasses were held for $48 \mathrm{~h}$ at $2{ }^{\circ} \mathrm{C}$. For years 1 and 2, the right side of the carcasses were ribbed between the 12 th and 13 th ribs and USDA yield and quality data were collected by an experienced grader. For year 3, carcasses were cut into quarters between the 13th rib and 1st lumbar vertebrae, placed into gondolas and shipped immediately by refrigerated truck to Nantahala Meats in Franklin, NC. Quarters were placed on the rail for $24 \mathrm{~h}$ and the forequarters were then ribbed between the 12th and 13th ribs and USDA quality and yield grade data were collected by the same experienced grader.

\section{Carcass Fabrication}

After grading, carcasses were fabricated into the following primals and subprimals and weights were recorded: brisket (NAMP 120), shoulder clod (NAMP 114), chuck roll (NAMP 116A), ribeye roll (NAMP 112A), mock tender (NAMP 116B), strip loin (NAMP 180 PSO 2), tenderloin (NAMP 189B), top sirloin (NAMP 184), knuckle (NAMP 167), 
inside round (NAMP 168), flat (NAMP 171B), eye of round (NAMP 171C), and flank steak (NAMP 193). After weighing the strip loin (NAMP 180 PSO 2), a $2.5-\mathrm{cm}$ steak was removed from the anterior end, vacuum-packaged, aged $14 \mathrm{~d}$, and frozen, for slice shear force analysis. For year 3, an additional $1.5-\mathrm{cm}$ steak was removed, trimmed free of epimysium, vacuum-packaged, and frozen for subsequent determination of proximate composition.

\section{Slice Shear Force Evaluation}

Steaks were removed from the packaging and a frozen weight was collected, then steaks were placed in the cooler to thaw at $2{ }^{\circ} \mathrm{C}$ overnight. The following morning, steaks were blotted dry and thawed weight was collected. Copper-constantan thermocouples attached to a potentiometer (Model No: 92000-00, Eutech Instruments Pte Ltd., Singapore) were placed in the approximate geometric center of the steak to record temperature. Steaks were then cooked on an electric grill (Model No: GR144, Salton Inc., Lake Forest, IL) until the internal temperature of $70{ }^{\circ} \mathrm{C}$ was reached. Cooked weight was recorded, along with time to reach final temperature, and the final cook temperature. The procedure of Shackelford et al. (1997) was used to determine slice shear force for the cooked steaks. The lateral end of the steak was removed and the remaining steak was placed in a sizing box where a second parallel cut was made $5 \mathrm{~cm}$ from the initial cut. The $5-\mathrm{cm}$ sample was then placed into a cutting box with two $45^{\circ}$ angled slots that line up with the muscle fiber orientation. A double-bladed knife was then used to make 2 parallel cuts simultaneously across the sample in order to produce a $5-\mathrm{cm}$ long and $1-\mathrm{cm}$ thick slice that ran parallel to the muscle fibers. The sample was reoriented to collect an additional $5-\times 1-\mathrm{cm}$ slice. Samples were then placed on an Instron Universal Testing Machine 3365 (Instron Limited, High Wycombe, UK) and slice shear force was measured when a $1.02-\mathrm{mm}$ thick blade, traveling $500 \mathrm{~mm} / \mathrm{min}$, sheared the slice perpendicular to the fiber orientation. The second sample was then sheared and slice shear force was averaged for the 2 slices.

\section{Proximate Analysis}

For proximate analysis, the $1.5-\mathrm{cm}$ samples were thawed overnight at $2{ }^{\circ} \mathrm{C}$, powder homogenized in liquid nitrogen, and the powdered samples were refrozen for subsequent analysis.

Crude protein was determined using a Nitrogen Analyzer (Model No: FP-628, LECO Corp., ST.
Joseph, MI). Powdered samples were weighed and $\mathrm{N}_{2}$ content was determined by combustion. Crude protein was calculated as the nitrogen $\% \times 6.25$.

For moisture and fat determination (total lipid), a sample (approximately $2.0 \mathrm{~g}$ ) was weighed and sealed in an ANKOM bag. Samples were placed in a drying oven (Model No: 1350 FM, Sheldon Manufacturing Co., Cornelius, OR) at $100{ }^{\circ} \mathrm{C}$ overnight, cooled in a desiccator for $10 \mathrm{~min}$, and then weighed to determine moisture loss of the sample. Dried samples were then placed in the Crude Fat Extractor (Model No: ANKOM XT15, Ankom Technology, Fairport, NY). Following ether extraction, the samples were placed in a drying oven (Model No: 1350 FM, Sheldon Manufacturing Co., Cornelius, OR) at $100{ }^{\circ} \mathrm{C}$ for $15 \mathrm{~min}$ to remove any residual ether. Samples were then removed from the oven, cooled in the desiccator for $10 \mathrm{~min}$, and weighed to determine the crude fat content of the samples.

\section{Statistical Analysis}

A randomized complete block design was used to analyze the effect of selection for RADG and MARB in a replicated $2 \times 2$ factorial arrangement of treatments. RADG line and MARB line were analyzed for main effects and their interaction. This resulted in four treatment combinations: high RADG, high MARB (Hi/Hi); high RADG, low MARB (Hi/Lo); low RADG, high MARB (Lo/ $\mathrm{Hi}$ ); and low RADG, low MARB (Lo/Lo). Data were analyzed using GLM procedures in SAS 9.4 (SAS Inst. Inc., Cary, NC). Sire was considered a random variable and the effect of sire nested within the interaction of RADG and MARB (SIRE(RADG*MARB)) was used to test the main effects of RADG and MARB and their interaction. YEAR was analyzed as a replicate. Least squares means were calculated and separated using the LSD procedure of SAS 9.4 (SAS Inst. Inc., Cary, NC).

\section{RESULTS AND DISCUSSION}

Table 2 lists the EPD values for the sire treatment groups used in the study at the time of selection. Steer age at weaning (Table 3) was not affected $(P>0.60)$ by either RADG or MARB EPD selection. Additionally, steer weaning weights were not different $(P=0.78)$ across RADG EPD groups; however, Hi MARB steers were $9.4 \mathrm{~kg}$ heavier $(P=0.02)$ at weaning than their AVG MARB counterparts. None of the ultrasound compositional measures collected at weaning differed 
Table 2. EPD values for Angus sire treatment groups based on high (Hi) or low (Lo) residual average daily gain (RADG) and high (Hi) or breed average (AVG) marbling (MARB) EPDs used at the time of selection

\begin{tabular}{lcccccccc}
\hline \hline & \multicolumn{7}{c}{ Angus EPD Averages $^{2}$} \\
\cline { 2 - 8 } Treatment $^{1}$ & BW & WW & YW & RADG & CW & Marb & REA & Fat \\
\hline Hi/Hi & 0.97 & 57.3 & 115.7 & 0.29 & 40.0 & 1.00 & 0.80 & 0.009 \\
Lo/Hi & 1.50 & 56.7 & 100.0 & 0.06 & 22.7 & 1.30 & 0.71 & 0.010 \\
Hi/AVG & 1.90 & 61.2 & 110.0 & 0.27 & 37.8 & 0.51 & 0.62 & -0.020 \\
Lo/AVG & 1.50 & 62.7 & 105.0 & 0.06 & 28.0 & 0.44 & 0.62 & 0.005 \\
\hline
\end{tabular}

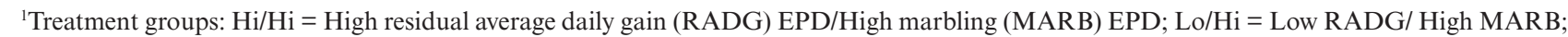
Hi/AVG = High RADG/ Low MARB; Lo/AVG = Low RADG/ AVG MARB.

${ }^{2}$ Angus EPD Averages: $\mathrm{BW}=$ birthweight; $\mathrm{WW}=$ weaning weight; $\mathrm{YW}=$ yearling weight $; \mathrm{RADG}=$ residual average daily gain; $\mathrm{CW}=$ carcass weight; Marb = marbling; REA = ribeye area; Fat = 12th-rib back fat.

Table 3. Effect of selection using high (Hi) or low (Lo) residual average daily gain (RADG) and high (Hi) or breed average (AVG) marbling (MARB) EPDs on growth performance and ultrasound body composition of Angus steers

\begin{tabular}{|c|c|c|c|c|c|c|c|}
\hline \multirow[b]{2}{*}{ Trait } & \multicolumn{2}{|c|}{ RADG EPD } & \multirow[b]{2}{*}{$P$-value } & \multicolumn{2}{|c|}{ MARB EPD } & \multirow[b]{2}{*}{$P$-value } & \multirow{2}{*}{$\frac{\text { RADG*MARB }}{P \text {-value }}$} \\
\hline & $\mathrm{Hi}$ & Lo & & $\mathrm{Hi}$ & AVG & & \\
\hline Weaning age, $\mathrm{d}$ & 228.7 & 228.8 & 0.96 & 228.2 & 229.3 & 0.66 & 0.40 \\
\hline Weaning weight, $\mathrm{kg}$ & 276.6 & 277.6 & 0.78 & 281.8 & 272.4 & 0.02 & 0.21 \\
\hline Weaning fat, $\mathrm{cm}$ & 0.38 & 0.41 & 0.31 & 0.42 & 0.36 & 0.05 & 0.92 \\
\hline Weaning ribeye area, $\mathrm{cm}^{2}$ & 52.3 & 52.0 & 0.79 & 53.9 & 50.3 & 0.01 & 0.07 \\
\hline Weaning IMF, \% & 3.8 & 4.2 & 0.02 & 4.1 & 3.9 & 0.07 & 0.13 \\
\hline Yearling age, day & 365.1 & 365.2 & 0.96 & 364.5 & 365.6 & 0.66 & 0.40 \\
\hline Yearling weight, kg & 414.2 & 408.2 & 0.35 & 413.3 & 409.1 & 0.51 & 0.22 \\
\hline Yearling fat, $\mathrm{cm}$ & 0.54 & 0.57 & 0.31 & 0.57 & 0.54 & 0.39 & 0.75 \\
\hline Yearling ribeye area, $\mathrm{cm}^{2}$ & 61.8 & 60.7 & 0.30 & 62.2 & 60.3 & 0.07 & 0.19 \\
\hline Yearling IMF, \% & 5.4 & 6.3 & 0.01 & 6.1 & 5.7 & 0.29 & 0.93 \\
\hline
\end{tabular}

$(P \geq 0.07)$ across RADG group, except for percentage IMF which was $0.4 \%$ higher $(P=0.02)$ in the Lo RADG compared with Hi RADG steers. This is likely due to the fact that 3 of the 4 bulls in the Lo/Hi category ranked in the first percentile of the Angus breed for marbling EPDs (data not shown). Weaning ultrasound $\mathrm{BF}$ and REA were $0.06 \mathrm{~cm}$ and $3.6 \mathrm{~cm}^{2}$ higher $(P \leq 0.05)$ in the Hi compared with AVG MARB sires, whereas percentage IMF tended $(P=0.07)$ to be $0.2 \%$ greater. The fat and REA increases with MARB selection were again most likely due to minor differences in the genetic values for the Hi compared with AVG MARB bulls (Table 2). Due to the low genetic correlations between marbling and BF and marbling and REA (Angus Journal, 2016), it would not seem logical to associate these differences with selection for increased marbling. Neither yearling weights nor compositional ultrasound measures differed across RADG or MARB selection groups, except for the Lo RADG steers having $0.9 \%$ higher $(P=0.01)$ percentage IMF compared with their Hi RADG counterparts.

Steers did not differ $(P>0.20)$ in on- or offtest weight across either RADG or MARB group
(Table 4). Gain $(P>0.70)$ and DMI $(P>0.23)$ did not differ between the RADG or MARB groups. The lack of differences in gain and feed intake across the RADG and MARB groups would suggest that feed efficiency did not differ; however, RFI was $0.54 \mathrm{~kg}$ lower $(P=0.05)$ in the Hi RADG steers compared with their Lo RADG counterparts (Table 4). The difference in RFI between the Hi RADG and Lo RADG steers is shown in Figure 1. Similarly, G:F was sighlty higher (0.01), although not significant $(P=0.11)$, in the Hi RADG compared with Lo RADG steers. The current findings for RFI, weaning and yearling weights, gain, DMI, and ADG concur with those of Koch et al. (1963) and Arthur et al. (2001) in that RFI measures appear to be independent of gain. Arthur et al. (2001) similarly found RFI and feed intake had a genetic correlation of 0.66 , whereas ADG had a correlation of 0.04 suggesting that feed intake can be reduced without affecting ADG. A low genetic correlation indicates that one trait can be manipulated without affecting the other; therefore, selection for feed intake appears to be less dependent of selection for ADG. This suggests that positive 
Table 4. Effect of selection using high (Hi) or low (Lo) residual average daily gain (RADG) and high (Hi) or breed average (AVG) marbling (MARB) EPDs on feedlot performance, feed intake, and feed efficiency of Angus steers

\begin{tabular}{|c|c|c|c|c|c|c|c|}
\hline \multirow[b]{2}{*}{ Trait } & \multicolumn{2}{|c|}{ RADG EPD } & \multirow[b]{2}{*}{$P$-value } & \multicolumn{2}{|c|}{ MARB EPD } & \multirow[b]{2}{*}{$P$-value } & \multirow{2}{*}{$\frac{\text { RADG }^{*} \text { MARB }}{P \text {-value }}$} \\
\hline & $\mathrm{Hi}$ & Lo & & $\mathrm{Hi}$ & AVG & & \\
\hline Start weight, kg & 401.5 & 392.8 & 0.22 & 396.8 & 397.4 & 0.93 & 0.70 \\
\hline End weight, $\mathrm{kg}$ & 552.9 & 542.6 & 0.28 & 547.9 & 547.6 & 0.98 & 0.67 \\
\hline Total gain, $\mathrm{kg}$ & 151.4 & 149.7 & 0.68 & 151.0 & 150.2 & 0.84 & 0.72 \\
\hline $\mathrm{ADG}, \mathrm{kg} / \mathrm{d}$ & 1.9 & 1.9 & 0.75 & 1.9 & 1.9 & 0.80 & 0.70 \\
\hline Total intake, $\mathrm{kg}$ & 1223.7 & 1262.1 & 0.22 & 1229.5 & 1256.3 & 0.39 & 0.30 \\
\hline Total DMI, kg & 897.8 & 925.2 & 0.23 & 901.9 & 921.1 & 0.39 & 0.33 \\
\hline Average intake, $\mathrm{kg}$ & 17.3 & 17.8 & 0.22 & 17.4 & 17.8 & 0.39 & 0.37 \\
\hline Average DMI, kg & 12.7 & 13.1 & 0.23 & 12.8 & 13.1 & 0.39 & 0.40 \\
\hline RFI, kg & -0.27 & 0.27 & 0.05 & -0.13 & 0.13 & 0.30 & 0.28 \\
\hline Gain:Feed & 0.17 & 0.16 & 0.11 & 0.17 & 0.16 & 0.24 & 0.11 \\
\hline
\end{tabular}

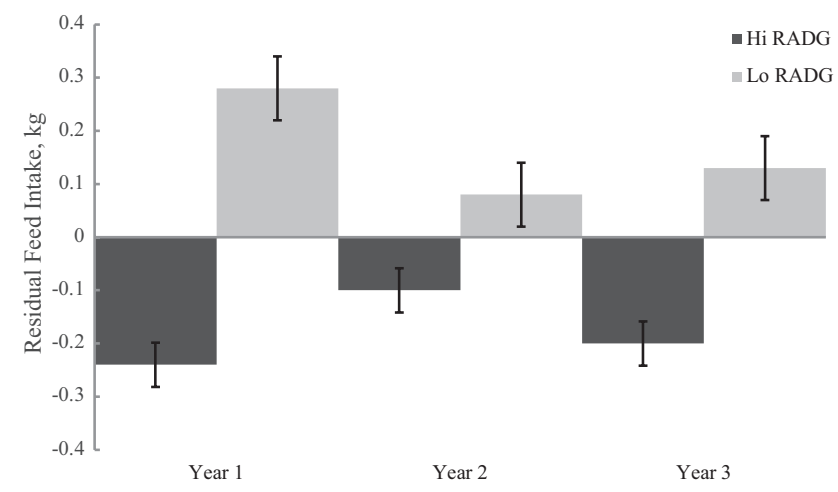

Figure 1. Residual feed intake $(\mathrm{kg})$ distribution in steers from cows bred to Angus bulls with high (Hi) and low (Lo) residual average daily gain (RADG) EPDs across the three years of the study.

selection pressure placed on the RADG EPD can improve overall production efficiency and growth above maintenance requirements, similar to RFI findings of Basarab et al. (2003). Actual intake of low RADG-selected steers was $0.39 \mathrm{~kg} / \mathrm{d}$ higher than high RADG (Table 4).

Steer age at slaughter (Table 5) was not affected $(P>0.40)$ by the either RADG or MARB selection. Additionally, steer slaughter weights were not different $(P=0.46)$ across MARB groups; however, Hi RADG steers were $18.5 \mathrm{~kg}$ heavier $(P=0.02)$ at slaughter than their Lo RADG counterparts. Likewise, steer hot carcass weights were not different $(P=0.87)$ across MARB groups, but HCW $(P=0.03)$ was $11.0 \mathrm{~kg}$ higher in the Hi RADG compared with Lo RADG steers. Even though there were differences in slaughter weight and $\mathrm{HCW}$, this did not translate to differences $(P>$ $0.50)$ in dressing percentage or yield grade, across RADG and MARB selection. The lack of difference in dressing percent and yield grade across the selection treatments concurs with the findings of Schenkel et al. (2004) and were expected due to the steers being slaughtered as they reached a back fat of approximately $1.3 \mathrm{~cm}$. Other studies have reported decreased fat levels and increased muscling in steers phenotypically sorted into low RFI (Hi RADG) groups compared with high RFI (Lo RADG) steers (Behrens et al., 2011).

There was an interaction $(P=0.05)$ between RADG and MARB for marbling score (Figure 2). The interaction resulted from the $\mathrm{Lo} / \mathrm{Hi}$ steers having a higher marbling score than all other treatment combinations. This is most likely due to the fact that the average MARB EPD for the Lo/Hi bulls ranked them in the top 1\% of the Angus breed, compared with the 8 th, 37 th, and 41 st percentile for the $\mathrm{Hi} / \mathrm{Hi}, \mathrm{Hi} / \mathrm{Lo}$, and Lo/Lo sires, respectively. Additionally, there were several bulls in the $\mathrm{Hi} / \mathrm{Hi}$ group whose MARB EPDs decreased -0.1 after selection, whereas several bulls in the Hi/Lo group had 0.06 increase in their MARB EPDs after selection (data not shown). A number of studies have shown that selection using marbling EPDs in Angus cattle generally results in increased marbling scores in the selected progeny (Gwartney et al., 1996; Sapp et al., 2002). Quality grade distribution for the AVG MARB group is as follows: high select $-2.2 \%$, low choice-18.5\%, average choice-25\%, high choice-32.6\%, low prime-20.7\%, and average prime $-1.1 \%$. In contrast, the QG distribution for the Hi MARB group is as follows: high select- $3 \%$, low choice- $6 \%$, average choice-22.2\%, high choice-31.3\%, low prime-25.3\%, and average prime-12.1\% (Figure 3). Lipid concentration of the longissimus was only determined in year 3 of the study (Table 5). Lipid percentage was $2.1 \%$ greater $(P=0.01)$ in the Lo RADG and Hi MARB compared with the Hi RADG and AVG MARB groups, respectively. These findings are consistent 
Table 5. Effect of selection using high (Hi) or low (Lo) residual average daily gain (RADG) and high (Hi) or breed average (AVG) marbling (MARB) EPDs on carcass traits of Angus steers

\begin{tabular}{|c|c|c|c|c|c|c|c|}
\hline \multirow[b]{2}{*}{ Trait } & \multicolumn{2}{|c|}{ RADG EPD } & \multirow[b]{2}{*}{$P$-value } & \multicolumn{2}{|c|}{ MARB EPD } & \multirow[b]{2}{*}{$P$-value } & \multirow{2}{*}{$\frac{\text { RADG*MARB }}{P \text {-value }}$} \\
\hline & $\mathrm{Hi}$ & Lo & & $\mathrm{Hi}$ & AVG & & \\
\hline Slaughter age, $\mathrm{d}$ & 547.8 & 542.4 & 0.46 & 542.5 & 547.7 & 0.47 & 0.88 \\
\hline Slaughter weight, kg & 592.4 & 573.9 & 0.02 & 580.5 & 585.7 & 0.46 & 0.58 \\
\hline Hot carcass weight, $\mathrm{kg}$ & 367.5 & 356.5 & 0.03 & 361.7 & 362.4 & 0.87 & 0.86 \\
\hline Dressing percent, $\%$ & 62.2 & 62.3 & 0.89 & 62.5 & 62.1 & 0.51 & 0.67 \\
\hline 12th Rib fat, cm & 1.1 & 1.2 & 0.34 & 1.2 & 1.1 & 0.22 & 0.12 \\
\hline Adj. 12th Rib fat, $\mathrm{cm}$ & 1.1 & 1.2 & 0.62 & 1.2 & 1.1 & 0.10 & 0.80 \\
\hline Ribeye area, $\mathrm{cm}^{2}$ & 78.6 & 77.1 & 0.30 & 78.2 & 77.4 & 0.56 & 0.27 \\
\hline $\mathrm{KPH}, \%$ & 2.1 & 2.0 & 0.57 & 2.1 & 2.0 & 0.36 & 0.17 \\
\hline Yield Grade & 3.2 & 3.2 & 0.86 & 3.2 & 3.2 & 0.58 & 0.19 \\
\hline Bone Maturity & 142.4 & 143.2 & 0.68 & 142.1 & 143.5 & 0.45 & 0.58 \\
\hline Lean Maturity ${ }^{1}$ & 147.9 & 147.9 & 0.99 & 147.8 & 148.1 & 0.91 & 0.64 \\
\hline Marbling Score ${ }^{2}$ & 596.8 & 631.0 & 0.14 & 632.2 & 595.6 & 0.10 & 0.05 \\
\hline Lipid Concentration, $\%$ & 6.6 & 8.7 & 0.01 & 8.6 & 6.8 & 0.01 & 0.83 \\
\hline
\end{tabular}

${ }^{1}$ Lean Maturity $100=$ A Maturity.

${ }^{2}$ Marbling Score $500=$ Modest, $600=$ Moderate.

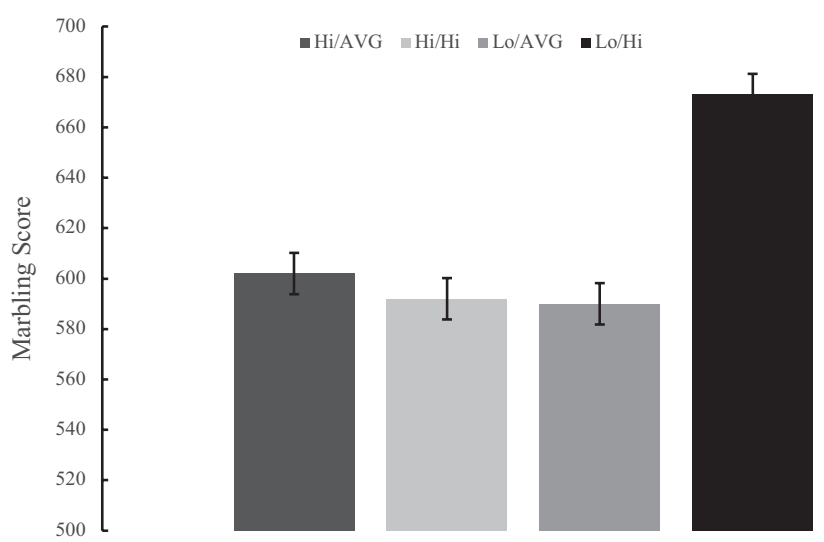

Figure 2. Marbling score in steers from cows bred to Angus bulls with high (Hi) or low (Lo) residual average daily gain (RADG) and high (Hi) or breed average (AVG) marbling (MARB) EPDs across sire selection category. Hi/AVG = High-residual average daily gain (RADG) EPD/breed average marbling (MARB) EPD; Hi/Hi = High RADG/High MARB; Lo/AVG = Low RADG/ breed average MARB; $\mathrm{Lo} / \mathrm{Hi}=$ Low RADG/High MARB. Marbling score is based on $500=$ Modest, $600=$ Moderate .

with the differences noted in marbling score across the RADG and MARB groups.

The major differences in the distributions of quality grade across MARB groups were that the AVG MARB group had higher percentages of Low Choice carcasses, whereas the Hi MARB group had higher percentages of Low and Average Prime carcasses (Figure 3). Over the course of this study, the numbers for low prime and average prime carcasses have increased in both the AVG MARB and Hi MARB selection categories (Figure 4). Marbling is considered to be a moderately heritable trait with heritability values of 0.35 (Arnold et al., 1991).
Hot side weights tended $(P=0.08)$ to be heavier in Hi RADG vs. Lo RADG carcasses by $4.5 \mathrm{~kg}$. As animals were harvested at a similar backfat thickness, this difference is likely related to increased time on feed. There was no RADG EPD selection effect $(P>0.14)$ on carcass subprimal weights (Table 6) with the exception of the mock tender $(P=0.01)$ and flat $(P=0.01)$ being heavier in the Hi RADG group than Lo RADG group and a tendency $(P=0.10)$ for the inside round to be heavier. When primal yields, as a percentage of hot side weight, were assessed, mock tender and flank yields were also $0.04 \%$ and $0.1 \%$ greater $(P=0.01)$ for the Hi compared with the Lo RADG groups (Table 7). Although the yields of these cuts were significantly different across RADG selection groups, the differences would have minimal impact on carcass value.

Weights (frozen, thawed, and cooked) of the longissimus steaks used for slice shear force determination did not differ $(P>0.62)$ across either RADG or MARB group (Table 8 ). There was also no difference $(P>0.17)$ for thaw loss, cook loss, and total loss across MARB selection. However, cook loss and total loss was $1.7 \%$ and $1.6 \%$ greater $(P=0.02)$ in Lo RADG compared with Hi RADG (Table 8). Slice shear force on the longissimus muscle was not affected $(P>0.45)$ by RADG or MARB selection, indicating that selection for either feed efficiency or marbling has minimal impact on meat tenderness.

Some of the unexpected findings in this project may be due to changes in the genetic values (EPDs) of the sires as greater amounts of information were 


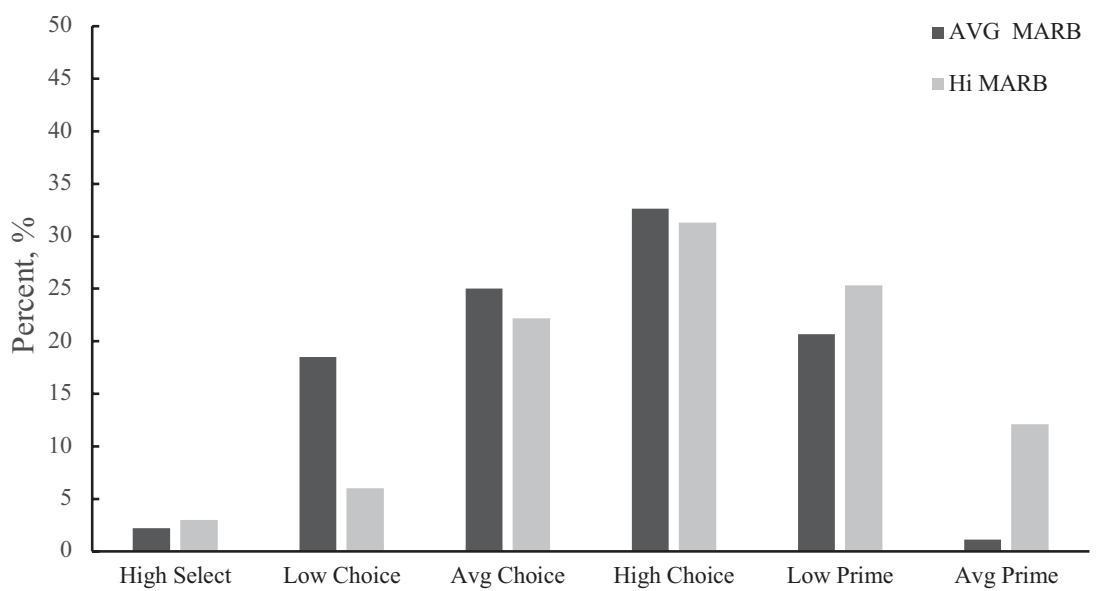

Figure 3. Carcass quality grade distribution in steers from cows bred to Angus bulls selected for high (Hi) and breed average (AVG) Marbling (MARB) EPD.
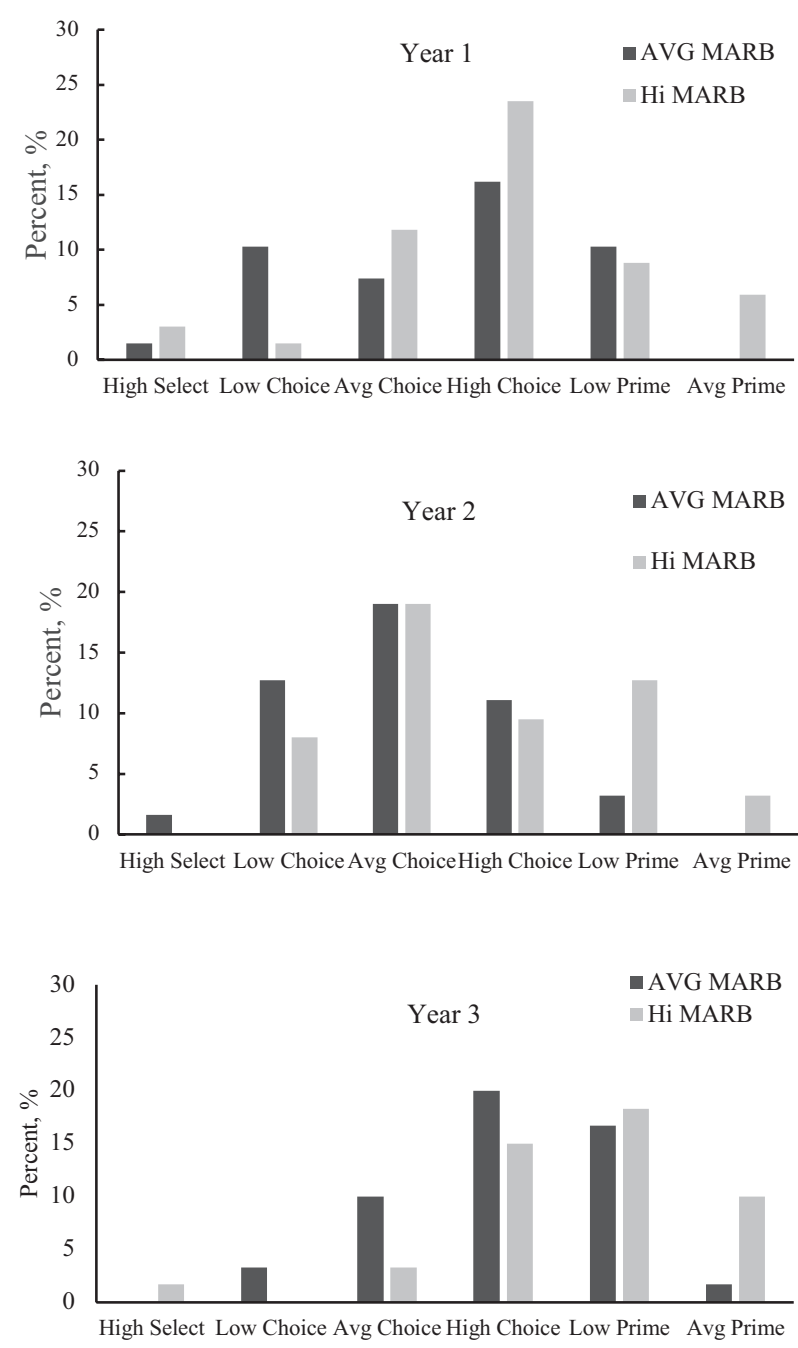

Figure 4. Carcass quality grade distribution in steers from cows bred to Angus bulls selected for high (Hi) and Breed Average (AVG) marbling (MARB) EPD across the three years of the study.

collected and evaluated. At the time of sire selection, the bulls used in this study ranked in the 5th percentile $(+0.27$ and higher) and 95th percentile (+0.10 and lower) for RADG EPD, with accuracies of 0.40 or higher. Over the last $3 \mathrm{yr}$, the number of calves with feed intake data submitted to the American Angus Association has increased resulting in more accurate descriptions of the bull's genetic values. For example, the RADG EPD's of two high RADG sires used in year one of the study have fallen to +0.26 and +0.28 , which places them in the 15th percentile of the breed. Similarly, RADG EPDs for 2 of the low RADG sires have increased to +0.16 and +0.14 , placing them in the 70 th percentile. In spite of the fact that the selection pressure applied to RADG in this study was not as great as initially thought, there was still a measured improvement in RFI after only $3 \mathrm{yr}$ of selection. Figure 1 shows the RFI distribution in steers for RADG EPD selection. Across the study, the variation between Hi RADG and Lo RADG EPDs suggests that there has been an improvement in Lo RADG compared with Hi RADG. In other studies, five generations of selection were required to see significant differences in RFI when using RFI phenotype (Low compared with High RFI) as the selection criteria (Donoghue et al., 2011). It is important to note that feed efficiency is only moderately heritable $\left(\mathrm{h}^{2}=0.36\right)$ in British breeds of cattle, so divergent selection resulting in significant changes in efficiency would not be expected in a single generation (Herd et al., 2003).

\section{CONCLUSIONS}

This 3-yr study supports the potential of the RADG EPD to be used as a selection tool to improve feed efficiency in beef cattle. Our results suggests that selection based on RADG EPDs will likely lower RFI and improve feed conversion ratio. More importantly, this increase in feed efficiency could be obtained without negatively affecting 
Table 6. Effect of selection using high (Hi) or low (Lo) residual average daily gain (RADG) and high (Hi) or breed average (AVG) marbling (MARB) EPDs on carcass primal and subprimal weights (kg) of Angus steers

\begin{tabular}{|c|c|c|c|c|c|c|c|}
\hline \multirow[b]{2}{*}{ Trait } & \multicolumn{2}{|c|}{ RADG EPD } & \multirow[b]{2}{*}{$P$-value } & \multicolumn{2}{|c|}{ MARB EPD } & \multirow[b]{2}{*}{$P$-value } & \multirow{2}{*}{$\frac{\mathrm{RADG}^{*} \mathrm{MARE}}{P \text {-value }}$} \\
\hline & $\mathrm{Hi}$ & Lo & & $\mathrm{Hi}$ & AVG & & \\
\hline Hot Side Weight & 182.6 & 178.1 & 0.08 & 180.7 & 180.1 & 0.78 & 0.49 \\
\hline NAMP 114 Shoulder Clod & 9.8 & 9.6 & 0.14 & 9.8 & 9.6 & 0.33 & 0.60 \\
\hline NAMP 116A Chuck Roll & 9.3 & 9.3 & 0.90 & 9.3 & 9.2 & 0.76 & 0.81 \\
\hline NAMP 116B Mock Tender & 1.2 & 1.1 & 0.01 & 1.1 & 1.1 & 0.74 & 0.25 \\
\hline NAMP 120 Brisket & 5.4 & 5.3 & 0.34 & 5.4 & 5.2 & 0.20 & 0.57 \\
\hline NAMP 112A Ribeye Roll & 5.0 & 4.9 & 0.59 & 5.0 & 5.0 & 0.61 & 0.62 \\
\hline NAMP 189A Tenderloin & 1.8 & 1.8 & 0.21 & 1.8 & 1.8 & 0.85 & 0.83 \\
\hline NAMP 180 PSO 2 Strip loin & 3.9 & 3.9 & 0.66 & 3.9 & 3.9 & 0.92 & 0.37 \\
\hline NAMP 184 Top Sirloin & 4.8 & 4.6 & 0.15 & 4.6 & 4.7 & 0.45 & 0.78 \\
\hline NAMP 167 Knuckle & 4.1 & 4.2 & 0.80 & 4.2 & 4.1 & 0.55 & 0.67 \\
\hline NAMP 168 Inside Round & 6.7 & 6.4 & 0.10 & 6.5 & 6.6 & 0.68 & 0.81 \\
\hline NAMP 171B Flat & 5.1 & 4.8 & 0.01 & 4.9 & 4.9 & 0.57 & 0.33 \\
\hline NAMP 171C Eye Round & 1.7 & 1.6 & 0.46 & 1.7 & 1.6 & 0.86 & 0.76 \\
\hline NAMP 193 Flank Steak & 0.7 & 0.7 & 0.21 & 0.7 & 0.7 & 0.78 & 0.38 \\
\hline
\end{tabular}

Table 7. Effect of selection using high (Hi) or low (Lo) residual average daily gain (RADG) and high (Hi) or breed average (AVG) marbling (MARB) EPDs on primal and subprimal yields as a percentage (\%) of hot side weight in Angus steers

\begin{tabular}{|c|c|c|c|c|c|c|c|}
\hline \multirow[b]{2}{*}{ Trait } & \multicolumn{2}{|c|}{ RADG EPD } & \multirow[b]{2}{*}{$P$-value } & \multicolumn{2}{|c|}{ MARB EPD } & \multirow[b]{2}{*}{$P$-value } & \multirow{2}{*}{$\frac{\text { RADG*MARB }}{P \text {-value }}$} \\
\hline & $\mathrm{Hi}$ & Lo & & $\mathrm{Hi}$ & AVG & & \\
\hline$\overline{\text { NAMP } 114 \text { Shoulder Clod }}$ & 5.4 & 5.4 & 0.79 & 5.4 & 5.3 & 0.30 & 0.96 \\
\hline NAMP 116A Chuck Roll & 5.1 & 5.2 & 0.23 & 5.2 & 5.1 & 0.85 & 0.85 \\
\hline NAMP 116B Mock Tender & 0.66 & 0.62 & 0.02 & 0.64 & 0.64 & 0.81 & 0.31 \\
\hline NAMP 120 Brisket & 2.9 & 2.9 & 0.83 & 3.0 & 2.9 & 0.18 & 0.80 \\
\hline NAMP 112A Ribeye Roll & 2.7 & 2.8 & 0.77 & 2.8 & 2.7 & 0.61 & 0.80 \\
\hline NAMP 189A Tenderloin & 1.0 & 1.0 & 0.99 & 1.0 & 1.0 & 0.87 & 0.92 \\
\hline NAMP 180 PSO 2 Strip loin & 2.1 & 2.2 & 0.26 & 2.1 & 2.1 & 0.84 & 0.52 \\
\hline NAMP 184 Top Sirloin & 2.6 & 2.6 & 0.43 & 2.5 & 2.6 & 0.19 & 0.41 \\
\hline NAMP 167 Knuckle & 2.2 & 2.3 & 0.24 & 2.3 & 2.3 & 0.60 & 0.44 \\
\hline NAMP 168 Inside Round & 3.7 & 3.6 & 0.26 & 3.6 & 3.7 & 0.45 & 0.51 \\
\hline NAMP 171B Flat & 2.7 & 2.6 & 0.01 & 2.7 & 2.7 & 0.14 & 0.23 \\
\hline NAMP 171C Eye Round & 0.93 & 0.94 & 0.66 & 0.94 & 0.94 & 0.95 & 0.91 \\
\hline NAMP 193 Flank Steak & 0.42 & 0.41 & 0.64 & 0.42 & 0.42 & 0.87 & 0.46 \\
\hline
\end{tabular}

Table 8. Effect of selection using high (Hi) or low (Lo) residual average daily gain (RADG) and high (Hi) or breed average (AVG) marbling (MARB) EPDs on cooking characteristics and slice shear force of longissimus lumborum strip steaks from Angus steers

\begin{tabular}{|c|c|c|c|c|c|c|c|}
\hline \multirow[b]{2}{*}{ Trait } & \multicolumn{2}{|c|}{ RADG EPD } & \multirow[b]{2}{*}{$P$-value } & \multicolumn{2}{|c|}{ MARB EPD } & \multirow[b]{2}{*}{$P$-value } & \multirow{2}{*}{$\frac{\mathrm{RADG}^{*} \mathrm{MARB}}{P \text {-value }}$} \\
\hline & $\mathrm{Hi}$ & Lo & & $\mathrm{Hi}$ & AVG & & \\
\hline Frozen Weight, g & 328.1 & 316.5 & 0.15 & 321.1 & 323.5 & 0.74 & 0.62 \\
\hline Thaw Weight, g & 320.9 & 309.9 & 0.17 & 314.1 & 316.7 & 0.72 & 0.73 \\
\hline Initial Temperature, ${ }^{\circ} \mathrm{C}$ & 7.5 & 7.8 & 0.34 & 7.7 & 7.6 & 0.68 & 0.49 \\
\hline Cook Weight, g & 267.7 & 253.5 & 0.06 & 258.1 & 263.1 & 0.49 & 0.44 \\
\hline Cook Time, min & 11.4 & 11.7 & 0.31 & 11.6 & 11.4 & 0.52 & 0.16 \\
\hline Thaw Loss, $\%$ & 2.2 & 2.1 & 0.70 & 2.3 & 2.1 & 0.82 & 0.06 \\
\hline Cook Loss, $\%$ & 16.4 & 18.1 & 0.02 & 17.7 & 16.8 & 0.19 & 0.17 \\
\hline Total Loss, $\%$ & 18.2 & 19.8 & 0.03 & 19.5 & 18.6 & 0.18 & 0.34 \\
\hline Visual Score $^{1}$ & 4.1 & 4.2 & 0.36 & 4.3 & 4.1 & 0.12 & 0.50 \\
\hline Slice Shear Force, $\mathrm{kg}$ & 17.9 & 17.7 & 0.69 & 18.0 & 17.6 & 0.46 & 0.63 \\
\hline
\end{tabular}

${ }^{1}$ Visual Score, $1-6 ; 1=$ very raw, $6=$ very well done. 
growth or carcass merit. Continued divergent selection for this trait should further improve feed efficiency and likely offer a model to enhance the understanding of the biological and physiological basis of feed efficiency in beef cattle.

\section{LITERATURE CITED}

Angus. 2016. Spring Sire Evaluation Report: angus trait heritablities and genetic correlations. Angus Journal. p. 390. Angus Productions, Inc, Saint Joseph, MO.

Arnold, J. W., J. K. Bertrand, L. L. Benyshek, and C. Ludwig. 1991. Estimates of genetic parameters for live animal ultrasound, actual carcass data, and growth traits in beef cattle. J. Anim. Sci. 69:985-992. doi:10.2527/jas.1991.693985

Arthur, P. F., J. A. Archer, D. J. Johnston, R. M. Herd, E. C. Richardson, and P. F. Parnell. 2001. Genetic and phenotypic variance and covariance components for feed intake, feed efficiency, and other postweaning traits in angus cattle. J. Anim. Sci. 79:2805-2811. doi:10.2527/ jas.2001.79112805

Arthur, P. F., and R. M. Herd. 2008. Residual feed intake in beef cattle. Rev. Brasileira de Zootecnia. 37: 269-279. doi:10.1590/S1516-35982008001300031

Basarab, J. A., M. A. Price, J. L. Aalhus, E. K. Okine, W. M. Snelling, and K. L. Lyle. 2003. Residual feed intake and body composition in young growing cattle. Can. J. Anim. Sci. 83:189-204. doi:10.4141/cjas.A02-065

Behrens, J. W., R. K. Miller, J. C. Bailey, J. T. Walter, A. N. Hafla, E. D. Mendes, D. S. Hale, T. Machado, L. O. Tedeschi, and G. E. Carstens. 2011. Effects of residual feed intake classification and breed type on carcass characteristics, tenderness and value in feedlot heifers. p. 761 . In: Proceedings of the Joint Annual Meeting of American Society of Animal Science and American Dairy Science Association, 89, New Orleans, LA. Federation of Animal Science Societies. [accessed May 7, 2019.]. http://www. jtmtg.org/JAM/2011/toc.asp.

Donoghue, K. A., R. M. Herd, J. F. Wilkins, and P. F. Arthur. 2011. Onset of puberty and early-life reproduction in Angus females divergently selected for post-weaning residual feed intake. Anim. Prod. Sci. 51: 183-190. doi:10.1071/an.10097

Gwartney, B. L., C. R. Calkins, R. J. Rasby, R. A. Stock, B. A. Vieselmeyer, and J. A. Gosey. 1996. Use of expected progeny differences for marbling in beef: II. Carcass and palatability traits. J. Anim. Sci. 74: 1014-1022.
Herd, R. M., J. A. Archer, and P. F. Arthur. 2003. Reducing the cost of beef production through genetic improvement in residual feed intake: opportunity and challenges to application. J. Anim. Sci. 81: E9-E17. doi:10.2527/ jas.2003.8113

Koch, R. M., L. A. Swiger, D. Chambers, and K. E. Gregory. 1963. Efficiency of feed use in beef cattle. J. Anim. Sci. 22: 486-494. doi:10.2527/jas. 1963.222486

Lancaster, P. A., G. E. Carstens, D. H. Crews, Jr, T. H. Welsh, Jr, T. D. Forbes, D. W. Forrest, L. O. Tedeschi, R. D. Randel, and F. M. Rouquette. 2009. Phenotypic and genetic relationships of residual feed intake with performance and ultrasound carcass traits in brangus heifers. J. Anim. Sci. 87:3887-3896. doi:10.2527/jas.2009-2041

MacNeil, M. D., N. Lopez-Villalobos, and S. L. Northcutt. 2011. A prototype national cattle evaluation for feed intake and efficiency of angus cattle. J. Anim. Sci. 89:39173923. doi:10.2527/jas.2011-4124

Nielsen, M. K., M. D. MacNeil, J. C. M. Dekkers, D. H. Crews Jr, T. A. Rathje, R. M. Enns, and R. L. Weaber. 2013. REVIEW: life-cycle, total industry genetic improvement of feed efficiency in beef cattle: blueprint for the Beef Improvement Federation. Prof. Anim. Sci. 29:559-565. doi.org/10.15232/S1080-7446(15)30285-0

Northcutt, S., B. Bowerman. 2010. Angus feed efficiency selection tool: RADG. In: Shauna H., editor. By the Numbers. Angus Journal. p.170-172. Angus Productions, Inc, Saint Joseph, MO.

Rolf, M. M., J. F. Taylor, R. D. Schnabel, S. D. McKay, M. C. McClure, S. L. Northcutt, M. S. Kerley, and R. L. Weaber. 2010. Impact of reduced marker set estimation of genomic relationship matrices on genomic selection for feed efficiency in angus cattle. BMC Genet. 11:24. doi:10.1186/1471-2156-11-24

Sapp, R. L., J. K. Bertrand, T. D. Pringle, and D. E. Wilson. 2002. Effects of selection for ultrasound intramuscular fat percentage in Angus bulls on carcass traits of progeny. J. Anim. Sci. 80:2017-2022. doi:10.1093/ansci/80.8.2017

Schenkel, F. S., S. P. Miller, and J. W. Wilton. 2004. Genetic parameters and breed differences for feed efficiency, growth, and body composition traits of young beef bulls. Can. J. Anim. Sci. 84(2): 177-186. doi:10.4141/cjas. A03-085

Shackelford, S. D., T. L. Wheeler, and M. Koohmaraie. 1997. Tenderness classification of beef. I. Evaluation of beef longissimus shear force at 1 or 2 days postmortem as a predictor of aged beef tenderness. J. Anim. Sci. 75: 2417-2422. 